\title{
Cloud Advection and Spatial Variability of Solar Irradiance
}

\author{
Joseph Ranalli \\ Penn State Hazleton \\ Hazleton, PA \\ jar339@psu.edu
}

\author{
Esther E.M. Peerlings \\ DLR Institute of Networked Energy Systems \\ Oldenburg, Germany \\ Esther.Peerlings@dlr.de
}

\author{
Thomas Schmidt \\ DLR Institute of Networked Energy Systems \\ Oldenburg, Germany \\ Th.Schmidt@dlr.de
}

\begin{abstract}
A model for predicting smoothing of solar irradiance by spatially distributed collectors was analyzed. The model assumed cloud advection dominates the relationship between sites and represented the distributed plant with a transfer function. The transfer function representing the smoothing effect was shown to be the Fourier transform of the plant's spatial distribution, and as such, the plant represents a low-pass filter. Comparison with measured data from the HOPE-Melpitz campaign showed that the model is able to replicate dynamics present in the measured plant transfer function and showed good frequency domain agreement. Generalization of the approach is needed for broader applicability, as the current analysis only validated against one-dimensional, advection dominated conditions. However, the approach warrants further study as it has demonstrated an ability to reveal frequency domain characteristics not currently reflected by state-of-the-art models.
\end{abstract}

Index Terms - variability, spatial aggregation, wavelet variability model, transfer function

\section{INTRODUCTION}

Expanded deployment of solar energy is a critical component of the worldwide response to climate change. Like other distributed forms of renewable generation, the rate of power delivery for solar photovoltaics (PV) is highly variable. This variability is generated power is rooted in variations in the input resource: received solar irradiance. Irradiance variability is caused both by predictable diurnal variations and more chaotic cloud and weather driven influences. Accurate forecasts of irradiance variability are important for electrical system operators, who need to balance generation and demand on a continuous basis. As a result, physical models and tools to predict the output from a PV system are of great practical interest.

Many large scale PV generation facilities and irradiance monitoring networks utilize point sensors for measurement of irradiance. It is well documented in the literature that the outputs of practical systems demonstrate smoothing relative to these single point monitoring measurements [1]. Aggregation of power at the utility-scale is important due to the relatively large capacities that can be involved. Typical utility-scale PV generation facilities might consist of a large number of PV panels distributed spatially over a local area in a nearly continuous fashion. On the other hand, aggregation of distributed PV generation at the transformer or substation level may also be of interest to grid operators, however, distributed PV typically consists of smaller installations distributed throughout a region or neighborhood in a more discrete fashion. As such, models that attempt to describe aggregation must address a diversity of plant shapes and distribution characteristics.

\section{A. Characterizing and Modeling Variability}

Previous modeling activities aimed at describing how spatial aggregation influences variability of the solar resource are abundant in the literature. A thorough review of the spatial and temporal nature of irradiance variability is given by Perez et al. [2], but a summary of models most relevant to the development in this study is provided in the subsequent paragraphs.

Variability is characterized using the change (ramp rate) of some physical parameter over a given time interval, $\Delta t$, and possibly considering a variable time integration period [3]. Perhaps the most typical physical parameter used for variability characterization is clear sky index, $K t^{*}$, because it normalizes out the effects of the diurnal cycle. Ramp rates of clear sky index may be represented by the symbol $\Delta K t_{\Delta t}^{*}$. Variability is then often characterized as the standard deviation of this term:

$$
\sigma\left(\Delta K t_{\Delta t}^{*}\right)
$$

An important study on quantifying spatial aggregation of irradiance was that of Hoff and Perez [1]. They considered the change of variability due to a network of collectors by considering the correlation (or lack of correlation) between individual sites in the network, showing that a network of uncorrelated sites causes a reduction in the variability scaling as $1 / \sqrt{N}$. They further describe correlation of sites in terms of cloud advection using a "dispersion factor", $D$, which is defined as the ratio between the length of the plant in the advective direction divided by the cloud velocity multiplied by the time interval (Eq. 2). This model, as the authors noted, was not yet generalized to consider arbitrarily distributed systems.

$$
D=\frac{L}{V_{c} \Delta t}
$$

Marcos et al. [4] describe the aggregation due to a spatially distributed plant in terms of a transfer function, $T F(f)$, between a single point measurement input and the entire site output. This promotes an interpretation of variability in a frequency domain context. They represent the transfer function 
as a first-order low-pass filter, whose single pole depends on the size of the plant. They apply this approach to model large dispersed plants [5]. The transfer function proposed takes the form of:

$$
T F(f)=\frac{K}{\frac{i f}{f_{c}}+1}
$$

where $i=\sqrt{-1}$. The cutoff frequency, $f_{c}$, was found to depend on the plant area in hectares, $S$, as follows:

$$
f_{c}=\frac{0.2}{\sqrt{S}}
$$

Another approach that focuses on frequency characteristics of the time series is used by the Wavelet Variability Model (WVM) of Lave et al. [6]. This model separates a clear sky index time series into discrete wavelet modes (i.e. frequency bands) and applies a frequency-dependent variability reduction factor that is a based upon the pairwise correlations between the distributed sites. Implementations of the WVM, such as that provided with the PVLIB MATLAB toolbox ${ }^{1}$, represent an arbitrarily distributed plant by creating a grid of individual points within the plant footprint. A centrally located reference site is intrinsic to this approach.

As the wavelet mode scaling is considered to be constant for a fixed plant distribution and cloud speed, the WVM approach also essentially consists of a fixed transfer function acting on the input time series. Individual wavelet modes in the WVM are scaled down based upon $\rho_{\text {sum }}$, the cumulative sum of all possible site-pair correlations within a given plant:

$$
w_{P}(t)=\frac{w_{\text {ref }}(t)}{\sqrt{\frac{N^{2}}{\rho_{\text {sum }}}}}
$$

Later studies by the same group [7] demonstrated that the individual pairwise correlation, $\rho$, between sites is directly related to the sites' separation distance and the dominant cloud transport speed. Specifically, they describe site pair correlation with an inverse exponential decay:

$$
\rho=\exp \left(-\frac{L}{\left(V_{c} / 2\right) \Delta t}\right)
$$

In the present study, we derive a one-dimensional cloud advection model (CAM), which follows the physical cloud advection approach of Hoff and Perez [1], and utilizes a transfer function representation as in [4]. This model specifically focuses on extending the approach used by Hoff and Perez to account for a variable plant spatial distribution.

\section{MOdELING APPROACH}

As in Marcos et al. [4], we represent the aggregating effect of the plant as a transfer function between a local irradiance time series at a single reference point, $g_{r e f}(t)$, and the aggregate irradiance averaged over the entire plant, $p(t)$. A transfer function describes this as the ratio of output to input,

\footnotetext{
${ }^{1}$ https://pvpmc.sandia.gov/applications/wavelet-variability-model/
}

albeit with each time series transformed into the frequency domain:

$$
T F(f)=\frac{P(f)}{G_{r e f}(f)}
$$

We define a one-dimensional spatial distribution of the collector, $d(x)$, to represent the density of collection per unit distance (units of $\frac{W}{m^{2} m}$ ). The distribution is normalized to the total spatial extent, $X$. Thus, the effective aggregate plant irradiance averaged over the full spatial extent can be represented as:

$$
p(t)=\int_{X} d(x) \cdot g(x, t) d x
$$

Following Hoff and Perez's cloud advection approach, the spatial dependence of the true local irradiance, $g(x, t)$, can actually be represented as the solar irradiance at the reference point, delayed by an advective time, $\tau=\frac{x}{V_{c}}$.

$$
g(x, t)=g_{r e f}\left(t-\frac{x}{V_{c}}\right)
$$

We rewrite the spatial coordinate of the plant's distribution in terms of this delay, and adopt the notation of $d^{*}(\tau)$. The aggregate irradiance then becomes a convolution between the plant's delay-coordinate distribution and the reference irradiance:

$$
p(t)=\int_{\frac{X}{V_{c}}} d^{*}(\tau) \cdot g_{r e f}(t-\tau) d \tau
$$

Because time-domain convolution represents multiplication in the frequency domain, substituting this into Eq. 7 yields the simple result that the transfer function representing the plant's spatial aggregation of irradiance is simply the Fourier transform of the plant's spatial distribution, as in Eq. 11. This result is similar to one previously derived to describe the dynamic response of flames to convected structures [8].

$$
T F(f)=\frac{D^{*}(f) \cdot G_{r e f}(f)}{G_{r e f}(f)}=D^{*}(f)=\mathcal{F}\left[d^{*}\left(\frac{x}{V_{c}}\right)\right]
$$

Given a transfer function, $T F(f)$, computed based on the plant's distribution, $d(x)$, at a given cloud speed, $V_{c}$, we can solve Eq. 7 for $P(f)$. Multiplying the transfer function by the input irradiance frequency spectrum, $G_{r e f}(f)$ yields the plant output. We can then perform an inverse Fourier transform to compute the desired aggregate time series, $p(t)$.

Note that this model operates directly on an irradiance time series as the input, $g_{r e f}(t)$, as opposed to the clear sky index time series used by the WVM in previous studies cited. As such, computing the clear sky index variability here would require the additional step of normalizing the simulated output by the predicted clear sky irradiance before calculating the ramp rates. 


\section{Model Results}

Though the formulation of this model is intended to describe an arbitrarily distributed plant, more detailed understanding of the modeling results may be developed by considering a simple plant geometry for which an analytical solution can be obtained. Consider a plant that can be represented as a uniform spatial distribution of length $L$, with a reference site at the front edge of the plant relative to cloud motion $(x=$ 0 ). The plant's distribution in the delay coordinate, $\tau$, is then represented by a difference of two Heaviside functions, $H$, the first switching on at a delay of 0 and the second at a delay of $\frac{L}{V_{c}}$. This results in a flat-top profile.

$$
d^{*}(\tau)=H(\tau-0)-H\left(\tau-\frac{L}{V_{c}}\right)
$$

Recognizing that the inverse of the time scale in Eq. 2 can be written as a frequency, we observe that the dispersion factor described by Hoff and Perez [1] is equivalent to the common nondimensional Strouhal Number encountered in fluid mechanics:

$$
S t=\frac{f L}{V_{c}}
$$

The Fourier transform of the uniform distribution profile given by Eq. 12 is then given by the complex analytical expression:

$$
T F(f)=\frac{1}{2 \pi i S t}[1-\exp (-2 \pi i S t)]
$$

Graphing the magnitude and phase of this transfer function results in a low-pass characteristic, representing the common sinc-in-frequency filter with time delay, as shown in Fig. 1. Values of zero in the magnitude occur at integer values of the Strouhal number, and are accompanied by a $+180^{\circ}$ shift in the phase. Excepting these $180^{\circ}$ shifts, the phase is characterized by a linearly decreasing trend, indicative of time delay, which can be quantified as in Eq. 15. This result matches natural intuition about the advective phenomena: the output is delayed by the time it takes for a cloud to advect across half the length of the overall plant.

$$
\tau_{\text {delay }}=-\frac{d \phi}{d \omega}=\frac{L}{2 V_{c}}
$$

Several characteristics inherent to this model (notably the high-frequency dynamics in magnitude that occur after the initial cutoff) are unique to the model used here, and do not occur in the WVM [6] or low-pass filter model by Marcos et al. [5], both of which basically just exhibit a decrease in magnitude with increasing frequency. Investigation of the advective physics at play reveals that these ringing phenomena occur due to the implicit assumption of perfect correlation of irradiance (albeit with delay) over the entire area of the plant. More specifically, the alternating high- and low- magnitudes observed in the TF at increasing frequency occur due to constructive and destructive interference associated with convolution of the cloud time series across the entire plant spatial distribution. In a sense, we can think of the zero magnitude value at a $S t=1$ as the two physical halves of the plant
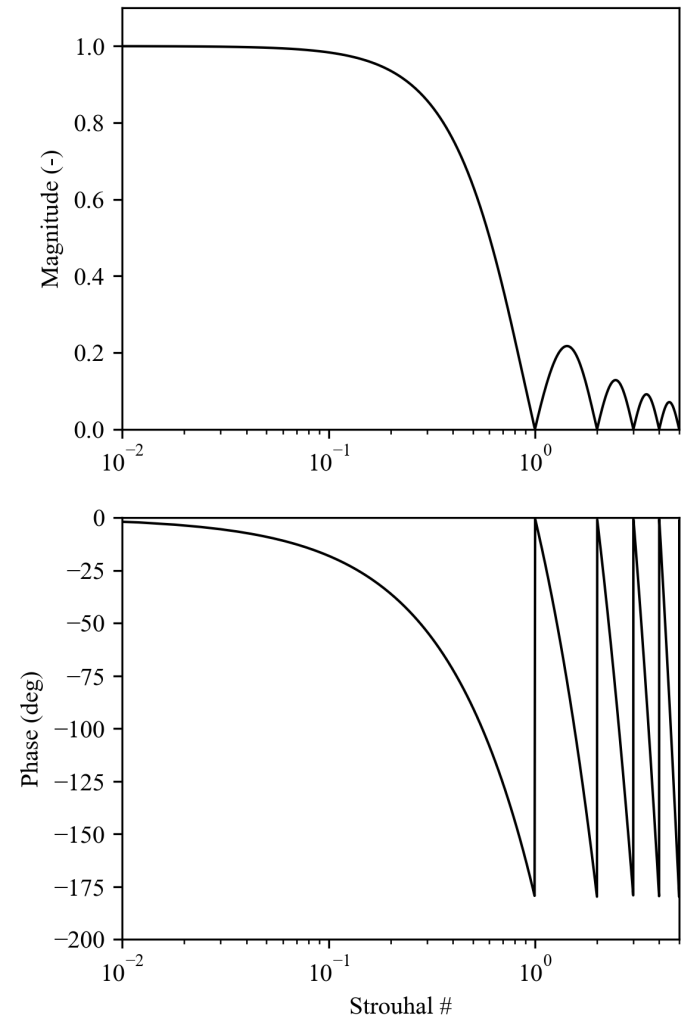

Fig. 1. Sample theoretical plant transfer function magnitude for a square shape plant. Frequency axis is nondimensionalized to represent Strouhal Number as in Eq. 13.

oscillating exactly out of phase at this frequency, resulting in a net zero oscillation when integrating. This effect could only occur if the distributed irradiance time series exhibits no noise at these frequencies (i.e. high spatial irradiance coherence). So in a sense, the presence of high frequency rebound in transfer functions provides the ability to discriminate cloud advection, and its presence in a real aggregate time series would be suggestive of a high degree of coherence at the relevant frequency, such that frequency dependent interference can be observed.

\section{A. Accounting for Reference Site Location}

Though the base form of this model describes a reference site at the leading edge (i.e. $\tau_{\text {delay }}=0$ ), the position of the reference site within the plant can be accounted for by rotating the angle (i.e. changing the delay) of the transfer function while maintaining the magnitude. We do so by multiplying the transfer function with a quantity with a magnitude of one and a phase angle that varies linearly with the frequency, as in Eq. 16. This is a well known operation in digital signals processing texts. Reference sites that lie upstream from the plant's front edge result in an increase in delay (negative $\Delta x$ ), while downstream corresponds to a decrease in delay. So in the example, shifting the reference site to be coincident with the center of the plant would result in a phase shift that produces 
zero time delay, indicating that the average position of clouds over the plant coincides with the reference measurement.

$$
T F_{\text {rot }}=T F \cdot \exp \left(2 \pi i f \frac{\Delta x}{V_{c}}\right)
$$

\section{B. Real Plant Transfer Function}

We tested the ability of the model to predict a transfer function for a real plant as well. We extracted the positions of an approximately co-linear set of measurement sites from the HOPE-Melpitz irradiance measurement campaign (described in more detail in subsequent sections). Each sensor was assumed to be an infinitesimal point, laid out in a linear south-to-north alignment. The distribution of these co-linear sensors is shown in Fig. 2. The reference site is assumed to be the central measurement site, with a location of $x=790 \mathrm{~m}$. The predicted CAM transfer function for this distribution was found by computing the Fourier transform of this plant distribution, as shown in Fig. 3.

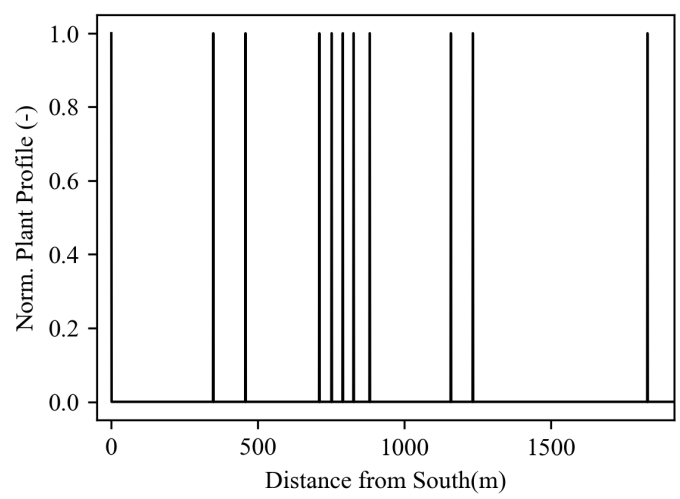

Fig. 2. Plant spatial distribution, $d(x)$ mapped into one-dimension for the full set of North-South points.

Similar to the simple uniform distribution case, the example transfer function in Fig. 3 remains a low-pass filter, with some rebounding high frequency dynamics visible after the initial cutoff. In this case, however, the irregular spacing of the measurement sites produces an uneven constructive/destructive interference pattern that results in several peaks, including the most significant around a frequency of $0.05 \mathrm{~Hz}$. No significant delay is evident in the transfer function phase, because of the centrally located reference site for this measurement. On the other hand, other dynamic response characteristics are present in the phase.

While observing the characteristics of a predicted transfer function is interesting academically, it is of much more practical interest to compare the response to real data.

\section{COMPARISON WITH MEASUREMENTS}

\section{A. Data Methodology}

To validate the model, we have utilized data from the HOPE-Melpitz measurement campaign outside Melpitz, Germany [9], which took place during September 10-27, 2013.
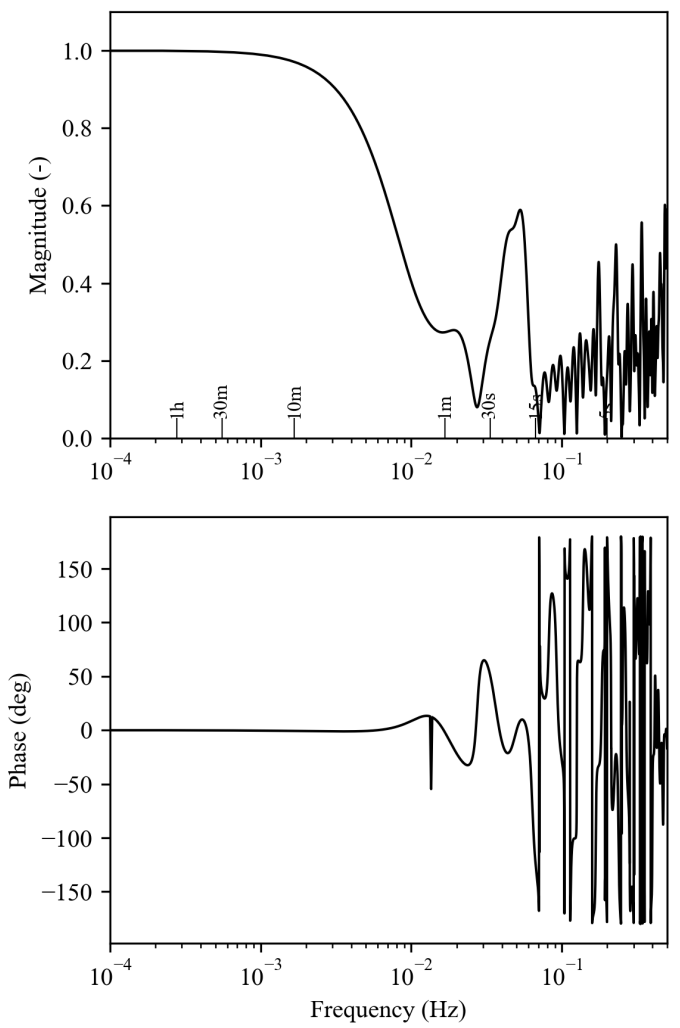

Fig. 3. Sample theoretical plant transfer function magnitude for all points indicated in Fig. 4, for time window A. Frequencies noted for some common time intervals.

The data from the campaign consists of 50 individual global horizontal irradiance (GHI) measurement sites, distributed over an approximately $3000 \mathrm{~m}$ x $2000 \mathrm{~m}$ region. Of these, 26 sensors were distributed in a dense $400 \mathrm{~m}$ x $200 \mathrm{~m}$ cluster in the center of the region, with the remaining 24 spaced more sparsely outside. An image of the campaign distribution is shown in Fig. 4. All measurements were of GHI time series with a $1 \mathrm{~s}$ resolution.

In order to test the 1-D model against results, we selected a subset of 11 sites aligned nearly linearly in a north-south direction (see Fig. 4). These are the same points that were described by the sample transfer function in Section III-B. Analytical methods for deriving the cloud motion vectors from a network of measurements are documented in the literature [10], [11]. However, for the purposes of this validation, two brief (around one hour) temporal windows were manually identified during which cloud advection along this north-south direction was evident in the time series. We identified these windows by finding periods where lags in the cross-correlation between the sensors (relative to a fixed reference) were consistent with a uniform cloud speed in this direction. A demonstration of this is shown in Fig. 5, which graphically demonstrates the increased site-pair cross-correlation that arises when advective lag is accounted for between multiple sites [2]. Details for the two windows studied are shown in Table I. 


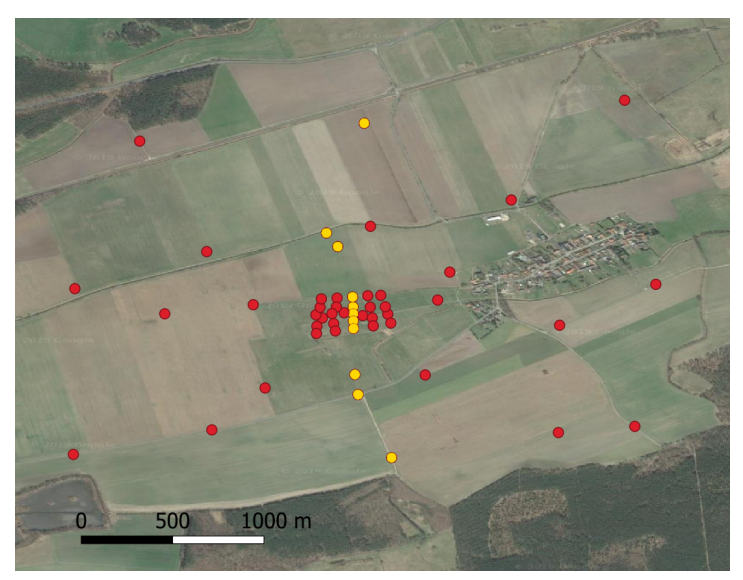

Fig. 4. HOPE-Melpitz measurement campaign layout. Yellow dots represent the subset of north-south oriented sites used for cloud advection. Image credit: Google and QGIS Contributors.

TABLE I

Cloud Advection Windows

\begin{tabular}{cccc}
\hline ID & Date & Times & Cloud Speed \\
\hline A & September 8, 2013 & $09: 15-10: 30$ & $20 \mathrm{~m} / \mathrm{s}$ to south \\
B & September 22, 2013 & $08: 45-09: 45$ & $30 \mathrm{~m} / \mathrm{s}$ to north \\
\hline
\end{tabular}

In all cases discussed in this validation, the reference site used was site 40 , which was the centrally located point within the measurement campaign footprint. The simulated plant output was computed by summing the time series from a set of measurement sites on the north-south alignment to simulate the output of the aggregation. Both the input and output time series were normalized to their mean to produce a transfer function with a low-frequency magnitude of unity. For both the input and output, Fast Fourier Transforms (FFTs) were computed and Eq. 7 was used to compute the transfer function for the real plant. Frequency domain averaging was performed to smooth the transfer function spectrum. Averaging was performed by splitting the overall window into 10 segments and applying a hanning window.

\section{B. Model Comparison}

In addition to simulation with the CAM as described in previous sections, other existing models were compared as well. The WVM was represented using the implementation included in the open source package pvlib python [12]. The model proposed by Marcos et al. was implemented using the analytical transfer function given in Eq. 3. As the plant modeled here is one-dimensional, no area is available for computation of the cutoff frequency (see Eq. 4), and so manual adjustment was used to empirically make the cutoff frequency match the approximate cutoff frequency of the CAM model. Output time series predictions for the CAM and Marcos et al. models were computed by applying the plant transfer function to the frequency domain representation of the reference point signal. As the WVM transfer function is dependent upon the input time series, frequency averaging (10 window segments with a hanning window) was performed similarly to for the simulated real data.

Fig. 6 shows a comparison of the plant transfer function as computed from the real data and for the three models. All three models predict the overall low-pass filter shape of the response. However, some of the more complex high frequency dynamics, including the peak around $0.05 \mathrm{~Hz}$, are only predicted by the CAM model. As stated previously, these dynamics are highly suggestive of advection-dominated physics, as correlation between sites is necessary to produce this interference pattern. The CAM model also reasonably matches the phase up to around a frequency of $0.06 \mathrm{~Hz}$ (a $\Delta t$ time period of around $15 \mathrm{~s}$ ). The WVM model basically avoids any phase dynamics, while the Marcos et al. model exhibits a single phase shift around the location of its pole, as is typical of first-order filter models. We also notice that coherence drops off significantly above the $0.06 \mathrm{~Hz}$ frequency, indicating that above this frequency, the dependence between the input and output is no longer well correlated.

Visual comparisons of time series do not always yield good measures of performance, but can be somewhat instructive. Time series of clear sky indices are shown in Fig. 7. The smoothing effect of aggregation is clearly visible between the reference and real cases. The WVM and CAM have comparable performance in replicating the time series, though qualitatively the CAM does represent some aspects of the overall shape somewhat better (e.g. around $50 \mathrm{~s}$, and $150 \mathrm{~s}$ ). The Marcos et al. model experiences some time shifting of the peaks relative to the reference signal, which is due to the phase mismatch induced by the single pole in the transfer function.

Comparisons of variability metrics are a more quantitative way to assess performance of the models, and are shown in Fig. 8. For this advection dominated time window, it can be seen that the WVM somewhat underpredicts the variability (overpredicts the smoothing), especially at increasing value of $\Delta t$. Both the Marcos et al. model and the CAM predict the variability and ROV relatively well for this time window.

\section{Changing the plant distribution}

As stated in the previous section, the high frequency dynamics observed in the transfer function magnitude are characteristic of strong spatial correlation between multiple measurement sites as advection takes place. To further confirm that these dynamics are well represented by the cloud advection model, we considered other selections of points from within the Melpitz footprint. Fig. 9 shows several such site subset selections, along with their corresponding transfer function magnitude comparisons. In each case, we see that though significant changes to the transfer function occur, the CAM does predict these changes to the major features of the dynamics. In all cases, the match begins to break down at higher frequencies, possibly associated with decreases in the coherence as described previously.

We perform a similar comparison for window B in Fig. 10. It is interesting to note that because the point selections for 

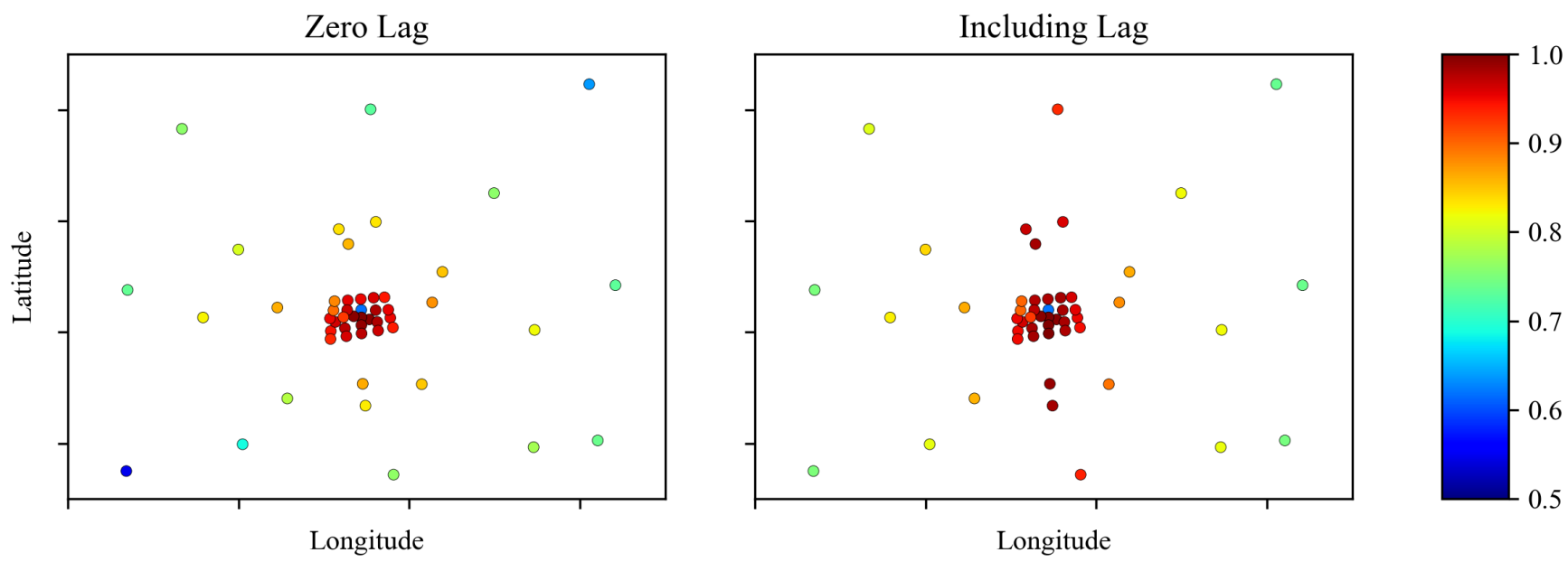

Fig. 5. Cross-correlation between sites and the central reference site. Left shows cross-correlation with zero lag, while right shows peak cross-correlation at any lag, demonstrating increased correlation corresponding to north-south cloud motion. Correlations are computed for Window A.
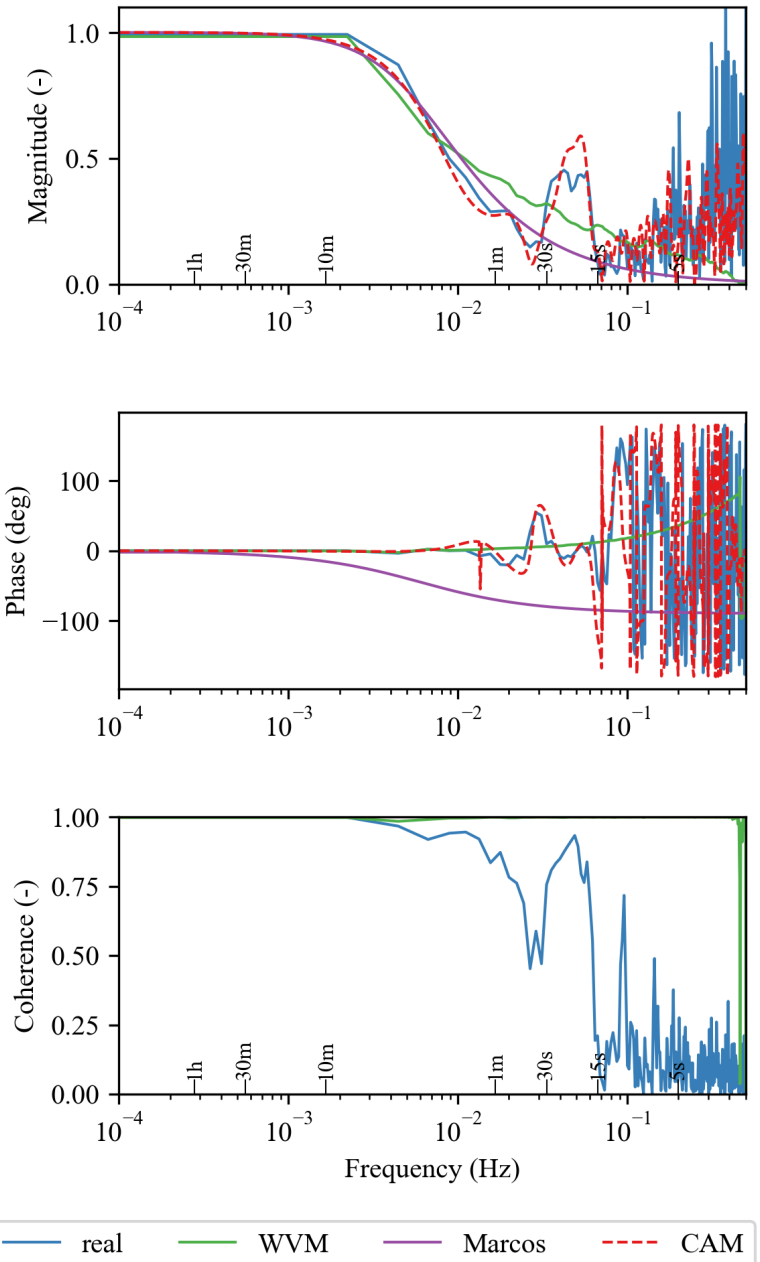

Fig. 6. Transfer function magnitude, phase and coherence for the case of all selected points indicated in Fig. 4 for window A. Frequencies noted for some common time intervals.
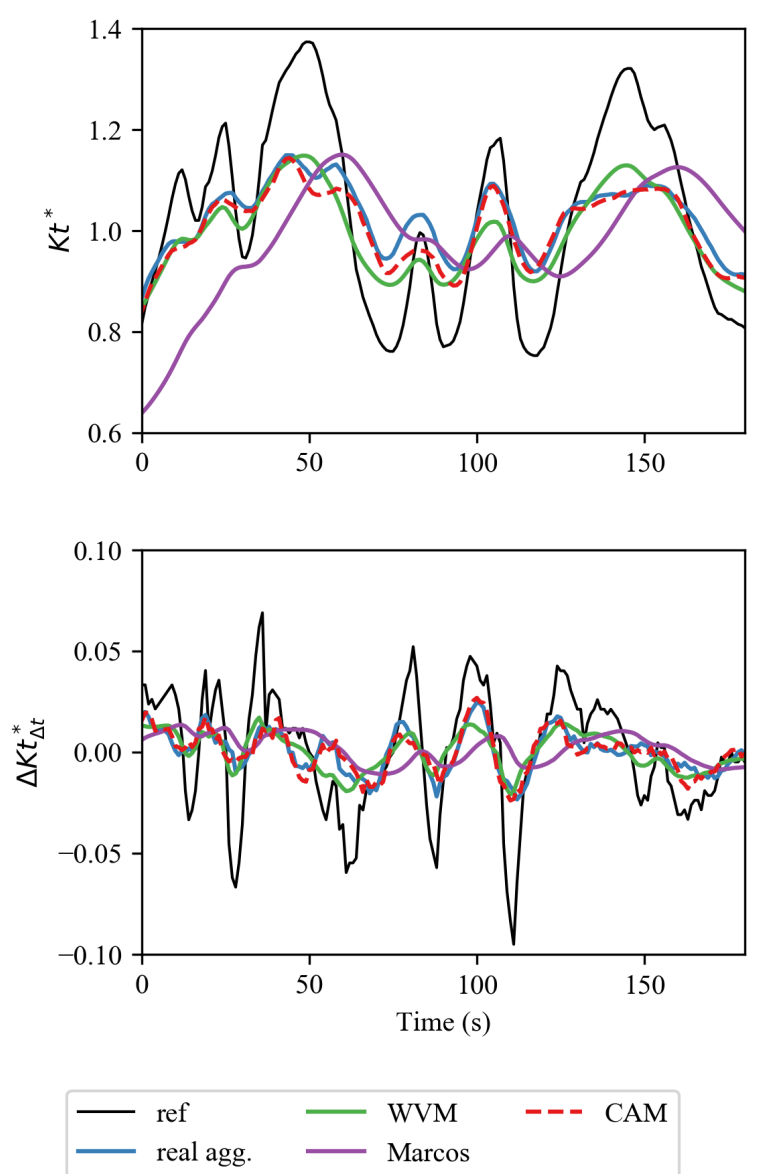

Fig. 7. Time series of $K t^{*}$ and $\Delta K t_{\Delta t}^{*}$ with $\Delta t=1 s$ for the case of all selected points indicated in Fig. 4 for window A. Compares the singlepoint reference, the real aggregate data, and results from three models. The reference point used is the central point in the domain. 

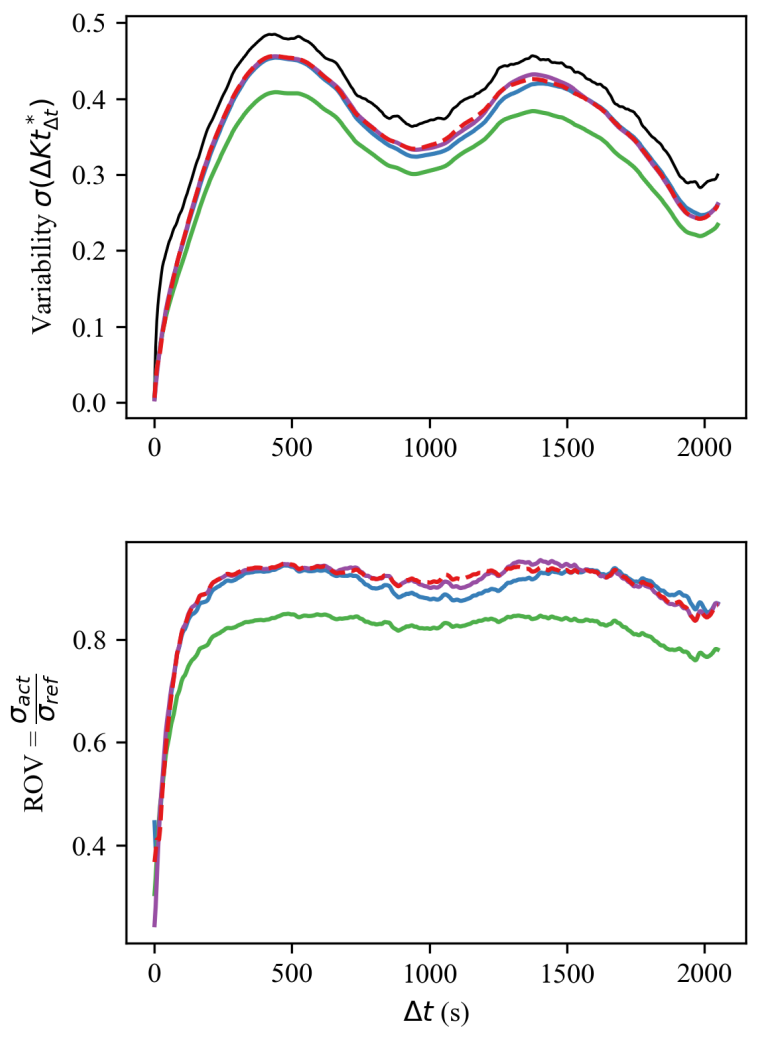

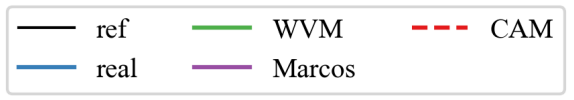

Fig. 8. Variability metrics as a function of $\Delta t$ for the real case and various models for the case of all selected points indicated in Fig. 4 for window A. ROV is the Relative Output Variability described by Hoff et al. [1], and is not shown for the reference case.

the four site subsets are the same between the Figs. 9 and 10, the predicted transfer functions for window $\mathrm{A}$ and window B are basically identical in shape. However, the window B transfer function is essentially stretched in frequency relative to window A, corresponding to the higher cloud velocity. This further supports the basic physics described by the Strouhal number scaling described in Fig. 1: higher cloud velocities correspond to higher cutoff frequencies.

The key finding from these results is that under cloud advection dominated conditions, like the two windows shown here, spatial aggregation can produce complex frequency response characteristics in the smoothed plant output, and that can be described by a simple physical model. These results also suggest that frequency domain analysis may be an important method for investigation of variability, because high frequency dynamics can be induced by aggregation. Though the CAM is relatively successful at producing good results in these time windows, its practical application in limited. As described in this paper, it has only been used to describe one-dimensional distributions of measurements (albeit with arbitrary shape), and only during carefully selected advection-dominated irra-
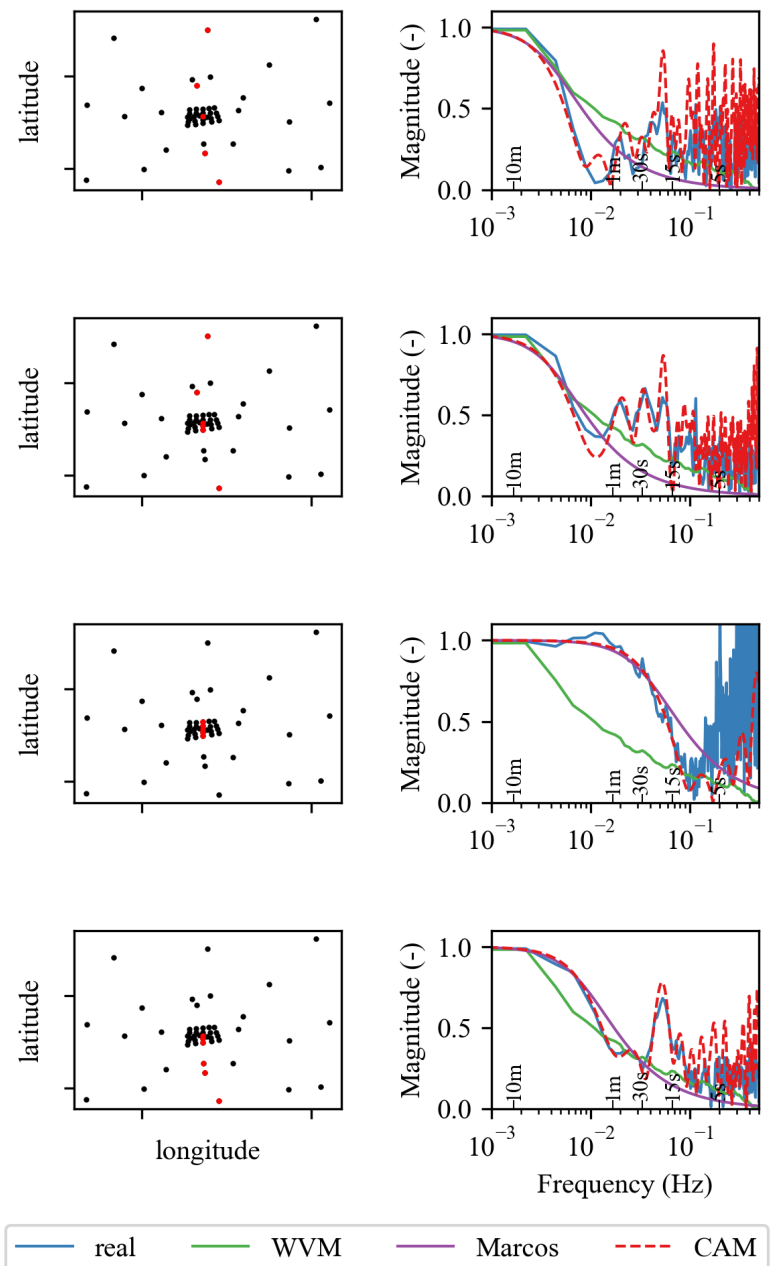

Fig. 9. Compared transfer function magnitudes (right column) for various selections of points (left column) for window A.

diance conditions. However, because these initial results show promise, further research is warranted to consider how this model could be applied to represent a two-dimesnional plant distribution, and how it could best be applied for more general cloud motion conditions.

\section{CONClusion}

The CAM analyzed in this study was developed to model the aggregation of irradiance by an arbitrarily distributed plant. The model was able to predict frequency domain behaviors of the plant transfer function that were observed in advectiondominated cloud conditions in a test dataset. Other models do not predict these dynamic behaviors, which is likely due to the fact that these models are developed to reflect the long-term variability reduction of distributed plants, for which site-pair correlation is much less consistent than in the limited cases tested here. In addition, the validation performed here was limited to a one-dimensional set of points, which do not really represent a typical distributed plant. 

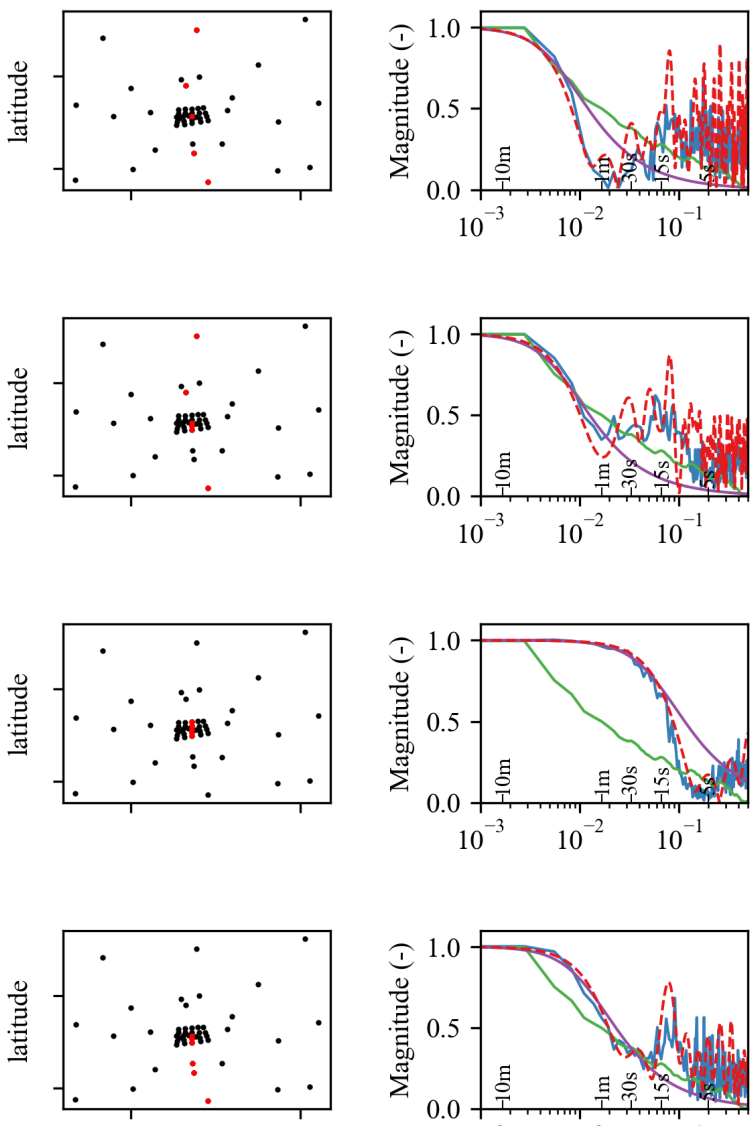

longitude

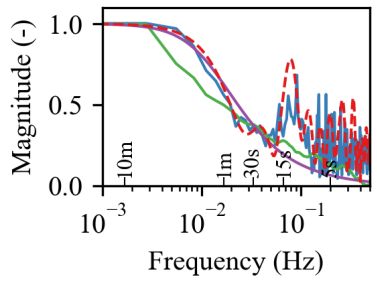

- real

real

WVM

Marcos

CAM

Fig. 10. Compared transfer function magnitudes (right column) for various selections of points (left column) for window B.

Though this study confirms that modeling of cloud advection is important to accurately representing a plant transfer function under short-term advection-dominated conditions, further work is needed to generalize for practical applications. Two-dimensional plants with multiple wind speeds, directions and cloud conditions must be considered. Additional work is also necessary to further describe the transition between the high spatial correlation behavior seen at low- to midfrequencies in the transfer functions here, and the reduction in coherence seen at higher frequencies. Nonetheless, analysis via transfer functions appears to be a promising approach to improve understanding of spatial smoothing of solar irradiance, and the approach used here may offer improved results when modeling variability under cloud advection conditions.

\section{ACKNOWLEDGMENT}

J.R. acknowledges financial support by Penn State Engineering Technology and Commonwealth Engineering and Penn State Hazleton, and appreciates the support of the Carl von Ossietzky University of Oldenburg and the DLR Institute for
Networked Energy Systems who hosted his visiting scientist appointment. Part of this work was supported by the Federal Ministry of Economic Affairs and Energy on the basis of a decision by the German Bundestag (project HyForPV, grant 0350032B).

\section{REFERENCES}

[1] T. E. Hoff and R. Perez, "Quantifying PV power Output Variability," Solar Energy, vol. 84, no. $10, \quad$ pp. 1782-1793, Oct. 2010. [Online]. Available: http://www.sciencedirect.com/science/article/pii/S0038092X10002380

[2] R. Perez, M. David, T. E. Hoff, M. Jamaly, S. Kivalov, J. Kleissl, P. Lauret, and M. Perez, "Spatial and Temporal Variability of Solar Energy," Foundations and Trends® in Renewable Energy, vol. 1, no. 1, pp. 1-44, Jul. 2016. [Online]. Available: https://www.nowpublishers.com/article/Details/REN-006

[3] G. M. Lohmann, A. H. Monahan, and D. Heinemann, "Local shortterm variability in solar irradiance," Atmospheric Chemistry and Physics; Katlenburg-Lindau, vol. 16, no. 10, pp. 6365-6379, 2016.

[4] J. Marcos, L. Marroyo, E. Lorenzo, D. Alvira, and E. Izco, "From irradiance to output power fluctuations: the PV plant as a low pass filter," Progress in Photovoltaics: Research and Applications, vol. 19, no. 5, pp. 505-510, Aug. 2011. [Online]. Available: https://onlinelibrarywiley-com.ezaccess.libraries.psu.edu/doi/10.1002/pip.1063

[5] J. Marcos, d. 1. Parra, M. García, and L. Marroyo, "Simulating the variability of dispersed large PV plants," Progress in Photovoltaics: Research and Applications, vol. 24, no. 5, pp. 680-691, 2016. [Online]. Available: https://onlinelibrary.wiley.com/doi/abs/10.1002/pip.2719

[6] M. Lave, J. Kleissl, and J. S. Stein, "A Wavelet-Based Variability Model (WVM) for Solar PV Power Plants," IEEE Transactions on Sustainable Energy, vol. 4, no. 2, pp. 501-509, Apr. 2013.

[7] M. Lave and J. Kleissl, "Cloud speed impact on solar variability scaling - Application to the wavelet variability model," Solar Energy, vol. 91, pp. 11-21, May 2013. [Online]. Available: http://www.sciencedirect.com/science/article/pii/S0038092X13000406

[8] J. A. Ranalli and D. Ferguson, "Convective wave motion during combustion instability in a low-swirl burner flame," in Western States Section of the Combustion Institute Spring Technical Meeting. Western States Section/Combustion Institute, 2010, pp. 11-19. [Online]. Available: https://pennstate.pure.elsevier.com/en/publications/convectivewave-motion-during-combustion-instability-in-a-low-swi

[9] A. Macke, P. Seifert, H. Baars, C. Barthlott, C. Beekmans, A. Behrendt, B. Bohn, M. Brueck, J. Bühl, S. Crewell, T. Damian, H. Deneke, S. Düsing, A. Foth, P. D. Girolamo, E. Hammann, R. Heinze, A. Hirsikko, J. Kalisch, N. Kalthoff, S. Kinne, M. Kohler, U. Löhnert, B. L. Madhavan, V. Maurer, S. K. Muppa, J. Schween, I. Serikov, H. Siebert, C. Simmer, F. Späth, S. Steinke, K. Träumner, S. Trömel, B. Wehner, A. Wieser, V. Wulfmeyer, and X. Xie, "The $\mathrm{HD}(\mathrm{CP})^{2}$ Observational Prototype Experiment (HOPE) - an overview," Atmospheric Chemistry and Physics, vol. 17, no. 7, pp. 4887-4914, Apr. 2017. [Online]. Available: https://www.atmos-chemphys.net/17/4887/2017/acp-17-4887-2017-discussion.html

[10] J. L. Bosch, Y. Zheng, and J. Kleissl, "Deriving cloud velocity from an array of solar radiation measurements," Solar Energy, vol. 87, pp. 196-203, Jan. 2013. [Online]. Available: http://www.sciencedirect.com/science/article/pii/S0038092X12003854

[11] J. L. Bosch and J. Kleissl, "Cloud motion vectors from a network of ground sensors in a solar power plant," Solar Energy, vol. 95, pp. 13-20, Sep. 2013. [Online]. Available: http://www.sciencedirect.com/science/article/pii/S0038092X13002193

[12] W. Holmgren, C. Hansen, and M. Mikofski, "pvlib python: a python package for modeling solar energy systems," Journal of Open Source Software, vol. 3, no. 29, p. 884, Sep. 2018. [Online]. Available: https://joss.theoj.org/papers/10.21105/joss.00884 